\title{
List of Distinguished Life Members of World Association of Laparoscopic Surgeons
}

A Mohamad Sayed Ahmad Alfaki (Qatar) A Yasemin Karageyim Karsidag (Turkey) Abayneh Admassu Abayneh (Ethiopia) Abdel Kato Sebbaale (Uganda) Abdel Rahman Hassan Abu Sabei (Qatar) Abdelhay Mohamed Ahmed Ali (Ireland) Abdelmonim Bashir Yagoub Ali (Sudan) Abdolmajid Ghaedizadeh (Iran) Abdolreza Pazouki (Iran) Abdul Haque Ansari (KSA) Abdul Kareem Jabbar Ghadhban (Iraq) Abdul Kato Sebbaale (Uganda) Abdul Sattar Paigham (Afghanistan) Abdullab Abdulwahid Salman (Iraq) Abdullab Sh.AM (Kuwait) Abel Alejandro Garibaldi (USA) Abhijit Biswas (India) Abhishek Kakkar (India) Abi Sukrisno (Indonesia) Abiye Hailu Gebre-Ab (Ethiopia) AboulMahsen (Saudi Arabia) Adeyeni Oladeji Laosebikan (Canada) Aditomo Widarso (Indonesia) Adnan Fatih Esen (Turkey) Ah See Ng (Malaysia) Ahmad Aurngzab (Pakistan) Ahmad Chammat (Saudi Arabia) Ahmad Nabil M Khafagy (Uganda) Ahmed Abdul-Khalik Zahab (KSA) Ahmed Alwan Kareem (Iraq) Ahmed Eltigani E Hussain (Ireland) Ahmed Fakhri Omer (Iraq) Ahmet Batki (Turkey) Ahmet Hamdy Dabash (KSA) Ahmet Hulusi Teksen (Turkey) Ahujakumar (India) Ajay Padmakar Chodhary (India) Alaa AK Mohammed (UAE) Alaa Mohsin Mohammad (Iraq) Alemseged Janka Dube (Ethiopia) Alexandre MS Roque Ambrosio (Portugal) Ali Mohammed Mahmnd (UAE) Ali Raza Brohi (Pakistan) Ali Riza Sozenoglu (Turkey) Alice M Salvador (Philippines) Alireza Khalaj (Iran) Allah AK Mohammed (UAE) Alparslan Baksu (Turkey) AM Abdulrahman Al-Zakari (UAE) Amaechi Francis Neozi (Nigeria) Amani Ahmed Mohamed Ali (UAE) Amardeep Singh Bansal (India) Aminur Rahman (India) Amir Abbas Manouchehri (Iran) Amir Vejdan (Iran) Amit Hindurao Sardesai (India) Amru Sofian (Indonesia) Anas Yousif Yakoob (India) Andhra Narasimha Reddy (India) Anisha Kellogg (India) Anita Roy (India) Anjali Kumar (India)
Anjila Yakalage Barreira Amaral (Portugal) Anolue Fredrick (Indonesia) Antonio Morries (Ghana) Antonio Valdes Morris (Ghana) Anurag Ramkrishna Nema (India) Aosama Rasheed Abdullah (Iraq) Araz Asi Mahmud (Iraq) Archana Dhaewan Bajaj (India) Archana Jain (India) Arie Adrianus Polim (Indonesia) AS Abdul Razak Al-Sweidan (KSA) AS Bansal (India)

Asha Sharma (India)

Ashees Lal Pravin (India)

Ashish Mody (India)

Ashon Sa’Adi (Indonesia)

Ashwini Gaur (UK)

Aswini Kumar Mishra (India)

Athar Ali (Tanzania)

Atul Sardana (India)

Avalapati Hari Prasad (India)

Aydin Kilinc (Turkey)

Aydin Kosus (Turkey)

Az Javed Ahmad (Pakistan)

Azhar Musa Al-Toriahi (Iraq)

Bansal Ramdas (India)

Basheer Anwer Bakar (Iraq)

Basima Shamkhi Jabar (Iraq)

Bassam Adil Ghazalah (UAE)

Bejoy Philip (India)

Belgin Fusun Selam (Turkey)

Benudhar Ray Ramsayer (India)

Bhamini Naresh Bhatia (India)

Bharti Jain (India)

Bharti Singh (India)

Bhaskar Joyti Paul (India)

Bhoopinder Jit Kaur (India)

Bhupesh Dayal (India)

Bhushan Kumar (India)

Bichhanda Krishna Mohanty (Botswana)

Bilal Mohid Baker (Jordan)

Billur Demiro Gullari (Turkey)

Birendra Kumar (India)

Brijesh Kumar Sharma (India)

Bulent Arici (Turkey)

Bulent Kars (Turkey)

Bulent Tekin (Turkey)

C Anthony Okonkwo (Nigeria)

Calderon Maria (Philippines)

Carlo Cajes Gurrea (Philippines)

Chandra Prakash Jain (India)

Chee Fah Chia (Taiwan)

Chetna Gulabani (India)

Chittapu Pardha Saradhi Rao (India)

Chittapu PSR Rauf (Republic of Maldives)

Christopher Ekwunife (Nigeria)

Cromwell HM Mwakirungu (Kenya)

Daljit Kaur Uppal (India)

Danish Javed (India)

David Kiragu (Kenya)

Deb Narayan Samanta (India)

Deepa Malhotra (India)
Deepa Srinivasan (India)

Deepak Chawla (India)

Deepak Kumar Gupta (India)

Deepak PJ Chan (China)

Deepak Singhal (India)

Deepakendu Mitra (India)

Demet Dikmen (Turkey)

Deniz Erturk Coskun (Turkey)

Derek PS Chan (Hong Kong)

Dhan Vikram Karkee (Nepal)

Dhananjay Kumar (India)

Dharam Paul (India)

Dharmendra Prabhat Sing Parmar (India)

Dheeraj Mulchandani (India)

Dutta Siva Sankar (India)

Duygu Isil Gencer (Turkey)

Duzgun Korkmaz (Turkey)

E Aleksamdrovna Serfeenkova (Russia)

Eatot Nyarumentene A Winarno (Indonesia)

Ebenezer Samuel Nkom (Nigeria)

Ebru Ozturk (Turkey)

Ebwell John RS (Indonesia)

Ekaterina Vasileva Kaloyanova (UAE)

Elif Meseci (Turkey)

Eman Mohammed kasim (Saudi Arabia)

Emel Turkoglu (Turkey)

Emre Erdogdu (Turkey)

Eser Evrim Eren (Turkey)

Esra Esim Bugukhayrak (Turkey)

F Ahmed Aly El-Fakharany (Saudi Arabia)

F Elrahman Elhag Edris Mohammed (UAE)

Fadhi Yaba Muhamed (Iraq)

Faiz Naqbool Fazili (India)

Farah Naaz (India)

Fatih Adanacloglu (Turkey)

Fatih Dogru (Turkey)

Fatima Zohra Ashraf (Turkey)

Fatma Nur Cetinkaya (Turkey)

Fawzia AM Jadallah (Dubai)

Firoz Khan MH Mohammed Haneefa (India)

Fransiska Mochtar (Indonesia)

Fuat Eserol (Turkey)

Gamal Samay Mahfouz (KSA)

Gamze Erdem (Turkey)

Gani Oladosu Salaudeen (Nigeria)

Gazala Khan (Pakistan)

Gazi Yildirim (Turkey)

Gazi Yildiz (Turkey)

Gede Widi Mariada (Indonesia)

Geetha Puliyath (India)

George Fanuel Jumba (Kenya)

Ghada Siddig Abdin Mohammed (Sudan)

Gheni Hashim Mohemmod (Iraq)

Girish Panday (India)

Gjayakrishnan (India)

Gokce Anik (Turkey)

Gokge Zeynep Uslu (Turkey)

Gokhan Sabah (Turkey)

Gokmen Iyigun (Turkey)

Gonca Yetkin Yildirim (Turkey)

Gorantla Lakshmi Narayana (India)

Gulsen Barcinli (Turkey) 
Gulten Gurgan (Turkey)

Gunjan Sabherwan (India)

Gupta Sib Sankar Mohapatra (India)

Hachem Juma Yasin (Iraq)

Hadi Touzandehjani (Iran)

Haider Abid Aun Ali Al Zubaidy (Iraq)

Haider Hasan (Iraq)

Hamid Abohari Sharifian (UAE)

Hamid Reza Tajari (Iran)

Hana Shaheed Kadim (UK)

Hani Amin Ismail (USA)

Hanom Husni Syam (Indonesia)

Hari Prasad Reddy Muppala (India)

Harish Bassi (India)

Hasan Majed Al-Dossary (KSA)

Hassan Hadi Yousif (Iraq)

Hassan Housaen Alhakeem (Syria)

Helmy Abdul Khalek Aboul Zahab (KSA)

Hemant Rakesh (India)

Henny Meitri (Indonesia)

Himmat Singh Rathore (India)

Hisham Ali Maghraby (UAE)

Hussain Khalifa Kadhem (Iraq)

Hussein Omar El-Bernawi (Libya)

Ibrahim Halil (Turkey)

Ibrahim M Hassen (Ethiopia)

Ibrahim Onur Ozen (Turkey)

Ichnandy Arief Rachman (Indonesia)

Iman Kamal Mallak (UAE)

Imtiaz Ahmed (India)

Indira Das (India)

Inu Mulyantoro (Indonesia)

Irfan Karaca (Turkey)

Isa Aykut Ozdemir (Turkey)

Isil Turan (Turkey)

Isin Fatma (Turkey)

Israa Mohsen Khalil (Iraq)

Jagpreet Singh Deed (India)

Jaibharathi Kundur Rudrappa (India)

Jaleel Hussien Hammoodi (Iraq)

Jameel Mohammed Salih (Iraq)

Jaspreet Singh Deed (India)

Jassim Ahmed Fakhro (Qatar)

Javad Saeid Faal (Iran)

Jayabalan Valliappan (Malaysia)

Jayahi Bala Juneja (India)

Jayasree Sundar (India)

Jimmy Yanuar Annas (Indonesia)

Jitendra Kumar Mangtani (India)

Jo-Ann B Badua (Philippines)

Joseph Eigbefoh (Nigeria)

Joseph S Shental (Israel)

Jude Okohue (Nigeria)

Juvvadi Srilatha (India)

Jyoti Gupta (India)

Jyotsna Rao (India)

Kabiri Homa (Afghanistan)

Kadan Kocatepe (Turkey)

Kadir Guzin (Turkey)

Kagan Kocatepe (Turkey)

Kailash Chandra Garg (India)

Kajla Bansal (India)

Kalingal Suja (India)

Kalpana Jindal (India)

Kalpana Kataria (India)

Kamal Abdel Gadir Mohamed (KSA)

Kandung Bowoleksono (Indonesia)

Kazim Qiamuzzama (India)

Kesavakrishna Brundavanam (India)

Khalaj Aligeza (Iran)

Khalaz ARMD (Iran)
Khaled Ahmad Kayed (UAE)

Khalifa Ali Al Jabri (Oman)

Khalil Yahia Mohamad (UAE)

Kiran Noshar Mevawalla (India)

Kirana Bala Dash (India)

Kishore Kumar Satpathy (India)

Koka Sreedevi (India)

Koray Ozbay (Turkey)

Krishna Mohan (USA)

Kulwant Rai (India)

Kunigal A Shivakumar (UK)

Kushum Sharma (India)

Lale Wetherilt (Turkey)

Lameck Zimba (Zambia)

Lateefa Abdulla Al Naeemi (UAE)

Latifa R Al Buainain (Baharin)

Latitha Kamini (India)

Laurent Marcel Hochart (Canada)

Lawa Hussein Abdulla (Iraq)

Leo Francis Nuval Aquilizan (Philippines)

Leonie-Martha Okaraga (Nigeria)

Lilly Kutty Benny (India)

Liqaa Reyadh Al-Khuzaee (Iraq)

Lovina SM Machado (Oman)

Luset Ince Koparan (Turkey)

Ma Ana Capucao Pangan (Philippines)

Madhu Sinha (India)

Madhulika Sinha (India)

Madhuri Prakash (India)

Magdi Ahmed Lolah (Egypt)

Magdi Genawi Ali (Ireland)

Mahavir Prasad Beniwal (India)

Mahesh Kumar Thakur (India)

Mahesh Man Pradhan (Nepal)

Mahesh Mishra (India)

Mahesh Prasad Khakurel (Nepal)

Mahinda Amarasiri Mallawatantri (Nepal)

Mahmoud Fellah Jahromi (Iran)

Mahmoud Honarmand (Turkey)

Mahmud Aurangzeb (Pakistan)

Makkar Mulkh Raj (India)

Manawor Ahsan (India)

Manoj Sukanraj Jain (India)

Mansour Amin Mohammd Al (Saudi Arabia)

Manvita Mahajan (India)

Maria Louroes Baricaua Escobar (Philippines)

Maria Nellbvezz C Candelario (Philippines)

Marie Victoria Gantiago (Philippines)

Marlieg Meshki (Iran)

Mary Anne Que Villarin (Philippines)

Mebratu Gember Tegesn (Ethiopia)

Meet Verm (India)

Mehmet Baki Senturk (Turkey)

Mehmet Guler (Turkey)

Mehmet Kaplan (Turkey)

Melek Buyukkinaci (Turkey)

Melike Batukan (Istanbul)

Mete Isikoglu (Turkey)

Michael Emefiele Aziken (Nigeria)

Minakshi Mishra (India)

Mohadmmad Nadeem Aslam (Pakistan)

Mohadmmad Rahim Bhurgri (Pakistan)

Mohamad Sabri B Hassan (Malaysia)

Mohamed Abdul Adim Elgabroun (Libya)

Mohamed Ali Hegazy (Saudi Arabia)

Mohamed Yehia Khalil (Qatar)

Mohammad Nadeem Aslam (Pakistan)

Mohammad Zarin (Pakistan)

Mohammd Abdul (Saudi Arabia)

Mohammd Ahmed Abdulla Eltom (Sudan)

Mohammd Ahmed Bella (Saudi Arabia)
Mohammed Hasan Jaafar (Iraq)

Mohammed Hussain (Iraq)

Mohd Niyaz (India)

Mohd Shafi Niaku (India)

Mohd. Omar Almoghanam (Saudi Arabia)

Mohd. Shakir Huwaish Alkoubaisy (UAE)

Mohini Raghvendra Goswami (India)

Mohmoud M Abdulrazek (Egypt)

Mohsen Aminpour (Iran)

Mohsin Hayder Kassar (Yemen)

Mokhtar Ali Mohamed Hassan (UAE)

Monika (India)

Mrinal Param Kinkar (India)

Muftah Abdulla Suwan (Libya)

Muhammad Abdullah Hussein (Iraq)

Muna Ahmed Al-Shehhi (UAE)

Munevver Hosgor (Turkey)

Munir Ahmad Rathore (United Kingdom)

Munirathnasri Nagappa (India)

Munther Moh. Adnan halabi (Syria)

Murat Mehmet (Turkey)

Muraveva Natalia (Russia)

Mustafa Mohd. Ramdam Bulugma (Libya)

Mutasim suleiman Daffalla (Ireland)

Muthukumarassamy Rajakannu (India)

Nada Abidalhur Alebrahimi (Iraq)

Nadia Tarak Barakat (Iraq)

Nadire Sevda Idil (Turkey)

Naimaa Khatoon Chaudhary (India)

Naimaa Khatoon Chaudhary (India)

Nand Kishore Prasad Singh (India)

Nandan Krishana Mohan (India)

Narender Kumar Parasher (India)

Nargis Harbans Sinjagil (KSA)

Natalia Muraveva (UAE)

Nausheen Fatma Mawal (India)

Navaneethan Sivarajasingam (Sri Lanka)

Nayantara Bijral (India)

Nazek Habeeb Al Salman (UAE)

Nazik Salameh Amarin (Jordan)

Needet Sver (Turkey)

Neeko Inees Chiriyankandath (India)

Neelam Vinay (India)

Neerja Sharma (India)

Neriman Basak Baksu (Turkey)

Nermin Kosus (Turkey)

Nese Yucel (Turkey)

Nicola Deeb Farah (Israel)

Nidal Jalal Arafat (Jordan)

Nilgun Kustarci (Turkey)

Nishat Mustafa Khan (Saudi Arabia)

Njila Yakalage Barreira Amaral (Portugal)

Nocola Deeb Farah (Israel)

Norman Oneil Machado (Oman)

Nzayisenga Jean Baptiste (Zambia)

Obote Wilson Williams (Uganda)

Oktay Erdener (Turkey)

Okunweme Ohiosimuan (Nigeria)

Oladapo Sunday Sotiloye (Nigeria)

Omar Abd Daim (Syria)

Omar Birol Durukan (Turkey)

Omar Eltigani Ibrahim (Sudan)

Omer Abbas Ramzi (Sudan)

Omertalipturhan (Turkey)

Omkar Prasad Sisodia (India)

Osama Elsayed Ammar (Saudi Arabia)

Osama Saied Algohary (KSA)

Ozgur Dogan (Turkey)

Ozlem Ozgur Gursoy (Turkey)

Oznur Gokcen (Turkey)

Pablo A. Candelario (Philippines) 
Padam Raj Pant (Nepal)

Pallavi Subhash Patil (India)

Pankaj Kumar Sinha (India)

Pankaj Malhrotra (India)

Parmalam_Ramanathan (India)

Parvinder Singh (India)

Parvinmohammadi (Iran)

Patel Morabhai Tushar (India)

Pierre C Lucien Charley Trevant (Haiti)

Pinar Yildiz (Turkey)

PN Sreeramulu (India)

Pola Farhad Husni (Iraq)

Ponifasio Mann (Nigeria)

Pooja Rajesh Vaswani (India)

Poonam Singh (India)

Poresh Boruah (India)

Prakash Kumar (India)

Prakash Kunan (India)

Pramod Singh (India)

Pran Singh Pugari (India)

Prasad Khakurel (Nepal)

Prashant Verma (India)

Pratibha Garg (India)

Predraq Urosevic (Belgradi)

Prem Kumar A (India)

Premal Priyakant Pancholi (India)

Prithvipal Rajaram Chavan (India)

Priti (India)

Priti Bajaj (Denmark)

Puliyath Geetha (Oman)

Pulkit Nandwani (India)

Puresh Boruah (India)

Pushpa Singh (India)

Putri Sri Lasmini (Indonesia)

Qasim Rashad Sleman (Iraq)

Raafat Raoof Ahmed Al-Turfi (Iraq)

Rabab Hosny Gabr (UAE)

Raed Ali Sharma (Turkey)

Rafat Nawaz (Bangladesh)

Rahime Beka (Turkey)

Raisan Mahdi Aljaberi (Iraq)

Raj Mulkh Makkar (India)

Rajasekhar Kanthaswamy (India)

Rajat Aashish Mody (India)

Rajeev Kumar (India)

Rajeev Mohan Seth (India)

Rajendra Mishra (India)

Rajendra Venkat Chivukula (India)

Rajesh Mittal (Sultanate of Oman)

Rajeshwar Prasad Mishra (India)

Rajeshwar Reddy (India)

Rajneesh Kumar Mishra (India)

Rajni Gupta (Kuwait)

Rakesh Hemant Shishodiya (India)

Rakha Aggarwal Tayal (India)

Rama Krishna Rao Boddu (India)

Ramadan Ali El-Gantri (Libya)

Ramesh Chandra Jain (India)

Ramesh Chandra Satpathy (India)

Ramesh L Kodaganallur (Australia)

Ratan Prasad (India)

Ratnasabapathy Pathmanathan (Sri Lanka)

Ravi Kant Arora (India)
Read Ali Sharaf (Turkey)

Rekha Thakur (India)

Riadhi Yulianto (Indonesia)

Richa Saini (India)

Rishi P Jain (India)

Ritu Kaushik (India)

Ritu Mogra (India)

Robbin Saikia (India)

Robert Sy Amaoo (Philippines)

Rukiye Agis (Turkey)

Ruperto, Jr, Gales Kisteria (Philippines)

Rupinder Kaur Ruprai (India)

Saad Dakhil F. Daraji (Iraq)

Sabaa Yehya Alkindialsayari (UAE)

Sabah Mahdi Al-Chawoosh (Iraq)

Sachin Hari Deshpande (India)

Sadook Naeem Nasaif (Iraq)

Sadullah Bulut (Turkey)

Safana Muslim Jaffar (Iraq)

Said IS Hassan (UAE)

Sajal Kumar (India)

Sajida Parveen Shaikh (Pakistan)

Sajira Bhasi (India)

Salah Ali Osman (KSA)

Salah Kamel Saksouk (UAE)

Salah Suliman Modawi (KSA)

Salem Ali Ahmed Al-Maashani (Oman)

Salem Nasser Alharethi (UAE)

Salma Omer Abdalla Ibrahim (Sudan)

Samrita De Banerjeee (India)

Samson Chandra (Indonesia)

Sana Abd Muslim (Iraq)

Sandeepa Vasudev (India)

Sanjay Kumar (India)

Sanjay Verma (Australia)

Sanjeev Kumar Jain (India)

Sarabjit Singh (India)

Sarika Jindal (India)

Satish Ramkrishna Sonawane (India)

Satrio Dwi Prasojo (Indonesia)

Scott Arockia Singh (India)

Sedat Evlioglu (Turkey)

Seema Theraja (India)

Sema Etiz Sayharman (Turkey)

Sema Kayatas (Turkey)

Serpil Telci (Turkey)

Sevim Turgut (Turkey)

Shafy Ali Khan SL (India)

Shahed Saeed Alzaabi (Germany)

Shahrokh Azimi Dehdezi (Iran)

Shailaja Chhetri (Sikkim)

Shakuntala Yadav (India)

Sheikh Md. Mahbubul Haque (Nigeria)

Shelar Sharad Shivdas (India)

Shreekrishna Shantaram Datye (Iceland)

Shyam Sunder Bansal (India)

Shyambahadur Prasad (India)

Siavash Falahatkar (Iran)

SK Leivon (India)

Snehal Vishnu (India)

Sonal Gupta (India)

Sonika Gupta (India)

Soo Nyung Kim (South Korea)
Sudhir Kumar (India)

Suja Kallingal (India)

Suliman Ali Elsheekh (KSA)

Suliman Mohamed Eldurrija (Libya)

Suman Lata Mendiratta (India)

Sumita Bachani (India)

Sumita Nayak (Kuwati)

Suphakarn Techapongsatorn (Thailand)

Suresh Kallianpur Baliga (UAE)

Suresh Kammiampur Baliga (Dubai)

Susanta Kumar Maiti (India)

Suseela Krishnan Kutty (India)

Sushrut Ashok Puranik (India)

Swodeep Mohanty (India)

Syarief Thaufik Hidayat (Indonesia)

Tabassum Ahmed (India)

Tahrir Abbas Ali Obaidy (UAE)

Tarek Hussein Mohd. Abu Ayad (Egypt)

Tegegn Gember Mebratu (Ethiopia)

Thabo Matsaseng (South Africa)

Tilahun Tesfayg Gizaki (Ethiopia)

Trisdian Tone Niaga (Indonesia)

Tsegazeab Kebede Kassaye (Ethiopia)

Tuba Gunay (Turkey)

Unal Turkey (Turkey)

Upasana Gupta (India)

Upsham Goyal (India)

Urmil Dhatarwal (India)

Usha Vishwanath (India)

Usman Abaidullah (Pakistan)

Vandan Kumar (India)

Vasileios Bagiokos (Greece)

Vasireddy VVSV Prasad (India)

Veerapol Khemarangsan (Thailand)

Vibha Bansal (India)

Vibhuti Kumar (India)

Vicenia Begui Balaso (Philippines)

Vicenia S Balajo (Philippines)

Vijay Bhusan Kalra (India)

Vijay Kumar Mishra (India)

Vijay Kumar Rajaram Naik (India)

Vijay Rajsinh Dilubha Gohil (India)

Vijay Ram Raje (India)

Vincent T Yu (USA)

Vinita Singh (India)

Vinod Kumar (India)

Vipin Nagpal (India)

Virochana Kaul (India)

Vishnu Jobanputra (India)

Vivek Gunvant Tank (India)

Vivek Khanna (India)

Vivek Sinha (United Kingdom)

Vurla Prabhavathi (KSA)

Wahyudi Gani (Indonesia)

Wilfred James Kimani (Kenya)

Wisam Hamza Abbas (Jordan)

Yasser Saleh Sabr (KSA)

Yeksin Helvacioglu Karatas (Turkey)

Yousry El-Sayed Abdu Aziz (KSA)

Yudi Mulyana Hidayat (Indonesia)

Zainab Hassan Al-Khafajy (Iraq)

Zeynep Akcig (Turkey) 